\title{
Restricted Hilbert transform for feasible light management
}

\author{
Muriel Botey*a, Waqas W. Ahmed ${ }^{\mathrm{b}}$, Ramon Herreo $^{\mathrm{a}}$ Ying Wu $^{\mathrm{b}}$, Kestutis Staliunas ${ }^{\mathrm{a}, \mathrm{c}}$, \\ aDepartament de Física, Universitat Politècnica de Catalunya (UPC), Barcelona, Catalonia; \\ b2Division of Computer, Electrical and Mathematical Sciences and Engineering, King Abdullah \\ University of Science and Technology (KAUST), Thuwal, Saudi Arabia; ' Institució Catalana de \\ Recerca i Estudis Avançats (ICREA), Barcelona, Catalonia
}

\begin{abstract}
A feasible restricted Hilbert Transform (HT) is presented to solve the challenging practical realization of non-Hermitian systems, restricting the complex susceptibility within practical limits. Beyond closed-conservative systems, the physics of non-Hermitian systems has become the playground to uncover unusual phenomena. Whilst Kramers Kronig relations break the temporal symmetry leading to causality, we proposed an analogous generalized Hilbert Transform (HT) to engineer complex media holding a nonistrotropic response, thus breaking the spatial symmetry. Applications of such HT range from tailoring the filed flows in arbitrary dimensions with particular application on VCSELS and edge-emitting lasers to cloaking arbitrary objects.
\end{abstract}

Keywords: Non-Hermitian Photonics, Metamaterials, PT-symmetry, Hilbert transform,

\section{INTRODUCTION}

Physical causality is closely related with the celebrated Hilbert Transform (HT) which warrants total "invisibility" of the future. In particular, in the case of optics such HT is well known as the Kramers-Kronig (KK) relations, connecting the real and imaginary parts of the permittivity a system. Indeed, the KK relations break the time symmetry in a way that it ensures that the response must occur at a later (not earlier) time of the arrival of its excitation. On the other hand, it is wellknown that nano-scale structured dielectric media, since the advent of the celebrated photonic crystals, may provide the momentum compensation for the control of over the scattering of waves. Yet, engineering the gain and loss profile instead of the refractive index uncovers analogous shaping effects [1]. However, only the interplay between both the refractive index and gain and loss modulations introduces unidirectionality in light management. Thus, non-Hermitian photonics has become one of the most fertile grounds in optics for a smart control of light, uncovering new physical mechanisms for the interaction between light and matter.

\subsection{Directionality fields to control the flow of light}

Most of the new intriguing features were initially proposed in a particular kind of such non-Hermitian systems, namely in those holding PT-symmetry [2][3]. An interesting extension of such broken-symmetry systems is when such unidirectionality in not homogeneous but depends on the spatial position [4]. Figure 1a represents a sinusoidal PTsymmetric media divided into two half spaces, where directionality follows the vector field forming a sink where and the field is expected to concentrate. Each half-space representing a generalization of the KK relations in space where the directionality of the field is warranted by the Fourier transform (zero in one half-space) of the final complex potential as indicated on Fig. 1 [5][6]. Recently, a generalized Hilbert Transform (HT) was proposed to design the complex permittivity to break the space directionality following arbitrary vector fields to tailor the flow of light [4] from any initial background potential, corresponding to a localized, spatially extended patterns, i.e. a periodic, quasiperiodic or randomly distributed background potential, see Fig. 1a. In the simplest 1D case, a scalar function $p(r)$ is sufficient, equal to $+1 /-1 \mathrm{for}$ right/left directionality, respectively to calculate the imaginary/real part of the refractive index from the real/imaginary counter as that suppresses either left or right scattering by the simple expressions:

$$
n_{R e}(x)=\frac{p}{\pi} P \int \frac{n_{I m}\left(x_{1}\right)}{x-x_{1}} d x_{1} ; n_{I m}(x)=\frac{-p}{\pi} P \int \frac{n_{R e}\left(x_{1}\right)}{x-x_{1}} d x_{1}
$$

*muriel.botey@upc.edu; donll.upc.edu 
Which correspond to a HT, and where the symbol $P$ means the principal value of the integral (the integral is calculated everywhere, except in at the singularity point).

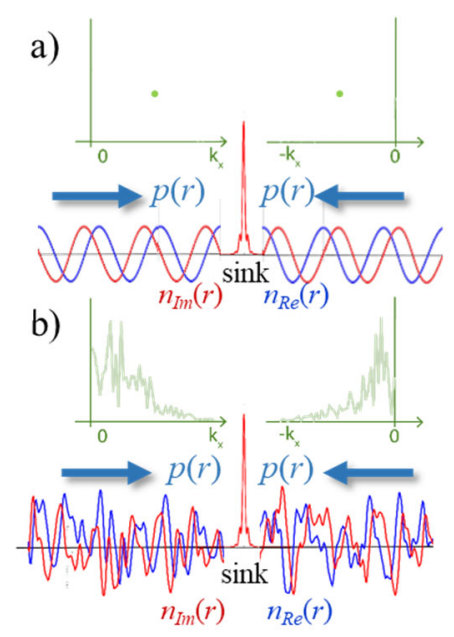

c)

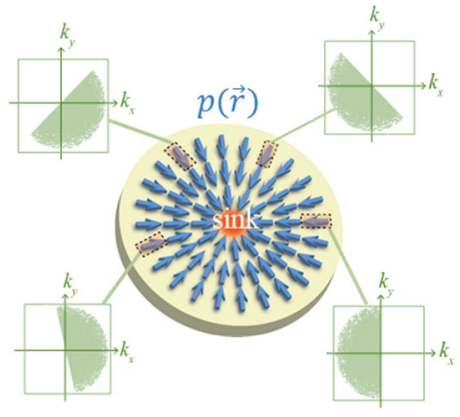

d)

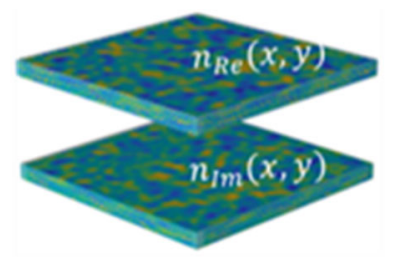

Figure 1. (a)/(b) One-dimensional periodic/random non-Hermitian potential consisting of two spatial domains of different parity, containing a sink. The first row represents Fourier spectra of each domain shown in the second row with given real, $n_{R e}(\mathrm{r})$ and imaginary part, $n_{I m}(\mathrm{r})$ (blue, and red, respectively) (c) Vector field, $p(r)$, to generate a complex directionality field in two spatial dimensions which may eventually create a sink for the probe field. The insets schematically represent the Fourier transforms of an arbitrary random directionality potential at several points. (d) Real, $n_{R e}(\mathrm{r})$ and imaginary part, and imaginary part, $n_{I m}(\mathrm{r})$ of the complex refractive index as calculated from the generalized HT.

Such directionality fields, may form a channel or create sinks for probe fields, see Figs. 1c and 1d. The HT expressed in eq. (1) can be generalized using non-homogeneous vector fields for which the HT has to be applied at each spatial position and to higher spatial dimensions [4].

\subsection{Generalized HT for invisibility on demand}

A similar procedure may be applied for an invisibility approach, i.e. to suppress the scattering of waves from/to particular directions and for particular frequency ranges. While the complete cancelation of a half-space of the Fourier transform of the complex refractive index completely cancels back-reflection, as shown in Figs 1a and 1b, it is also possible to suppress the scattering of waves from or to given directions and for particular frequencies, i.e., generating invisibility on demand. This is achieved when the modulation components within a limited invisibility area in $k$-space are set to zero, see Fig. $2 \mathrm{a}$, which uncouples incoming and outgoing wavevectors. Since a product in reciprocal space corresponds to a convolution in direct space, the Fourier transform of the area set to zero is the kernel of the convolution, in direct space, to be introduced in the generalized HT [7].

a)

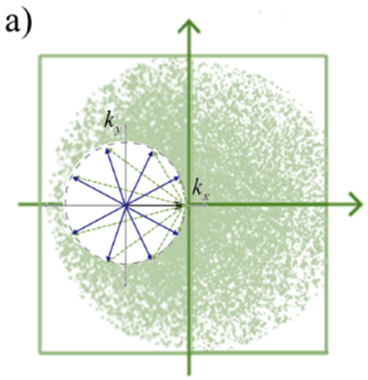

b)

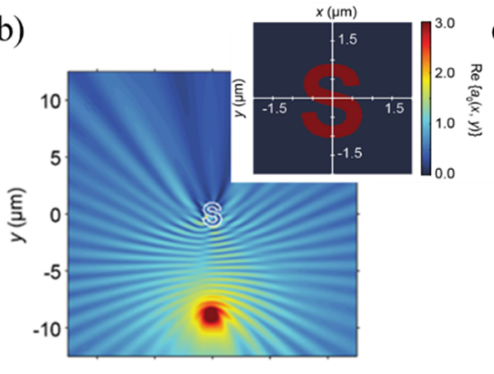

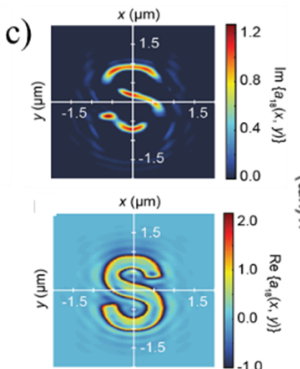

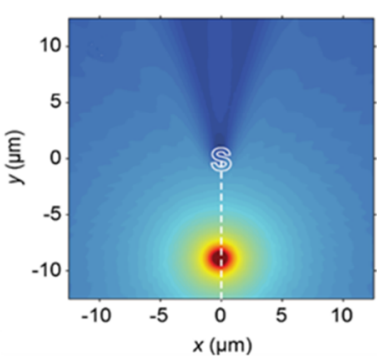

Figure 2. (a) Illustration of invisibility on demand; the modulation components set to zero within a limited invisibility area uncouple the scattering of incoming waves to reflected wavevectors. (b) Scattering simulation for the S-shaped object, with spatial refractive index $n_{R e}(\mathrm{r})$ distribution given in the inset, probed by a monochromatic point source. (c) Scattering 
simulation simulations for the modified S-shaped object with real, $n_{R e}(\mathrm{r})$ and imaginary part, and imaginary part, $n_{\operatorname{lm}}(\mathrm{r})$ of the complex refractive index provided in the inset.

Figure 1 shows the interference pattern between the incident and reflected waves from a dielectric S-shaped object when illuminated from a point-source. When the object is modified following the proposed scheme no interferences are observed in the simulation, see Fig. 2c. We note that in this scheme, a symmetric kernel allows producing purely dielectric "invisible" objects from two opposite directions.

In principle, the proposed HT would typically lead to media with optical gain, as for the case in Figs. 1d and 2c, and technical inconveniences might arise either for the fabrication of actual systems. The resultant complex index profile may demand a large number of actual materials for the practical realization due to the continuous variation of complex index values in space. Therefore, the fundamental question that arises is if the generalized HT following arbitrary directionality fields can also be implemented within a restricted (realistic) range of complex susceptibility of a material. It is possible to avoid such difficulties, by applying the HT iteratively.

\section{RESTRICTED HT}

\subsection{Dimensionality reduction}

We propose to introduce restrictions for the complex index values of the generalized HT, constraining them around certain particular values or confining them in some particular areas of the parameter space, without limiting the field unidirectionality or total invisibility. The proposal relies on an iterative procedure, to include such constraints to design the locally PT-symmetric systems with realistic index and gain/loss values.

To show the universality of the method to engineer the desired HT profiles in the restricted parameter space, we analyze distinct cases, which entail reducing the dimensionality in parameter space of complex refractive indices. Restricting the total filling factor of two materials leads to a $1 \mathrm{D}$ manifold in the refractive index in complex refraction index parameter space, while limiting the total filling factor of two materials to some upper bound results in a general 2D manifold of available complex refraction indices, a surface, see Fig. 4a. Another possibility is media constructed as a metamaterial build from a continuous family of size-scaled metachips in microwave range which restricts the complex refraction index space to a ring, as in Fig. 4b. Finally, another possibility is using a "poor man" collection of the discretely size-scaled resonators (for instance, of Helmholtz resonators in acoustics, or microwave metachips), corresponding to a discrete set of points in the complex index space see, Fig 4.c. All these restrictions involve a dimensionality reduction of the three proposed restrictions: $2 \mathrm{D} \rightarrow 2 \mathrm{D}, 2 \mathrm{D} \rightarrow 1 \mathrm{D}$ and $2 \mathrm{D} \rightarrow 0 \mathrm{D}$.

a)

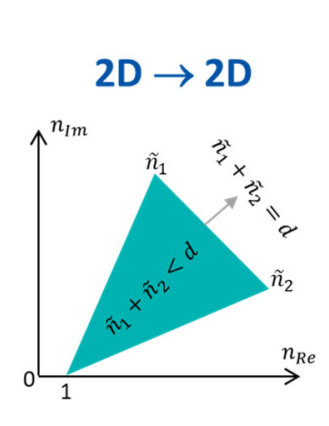

b)

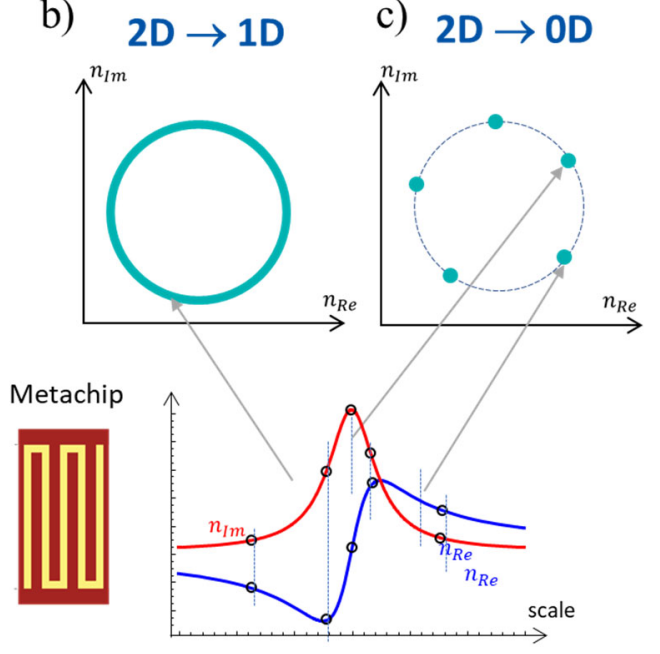

Fig. 4 Restrictions of the local HT for different restriction dimensionalities: (a) mixture of two different materials restricting the maximum density determines an area, (b) continuum of scalable metachips gives an available ring in n-complex space, and (c) limited collection of scaled-in-size meta-atoms gives a set of points in complex space (five points in this case). 


\subsection{Results}

To implement the proposed schemes, we apply an iterative procedure. The generalized HT with non-homogeneus directionality made on an arbitrary background potential to create desired directionality requires a large area of real/imaginary values of the complex refractive index. After performing the HT, we shift the unallowed complex layinf out of the restriction area. This process may break the HT, therefore the HT is again performed. And the procedure is iterated until the index values converge. The procedure may be characterized by a correlation coefficient for the generated potentials with different restriction dimensionalities that assesses the convergence of the restricted HT. Indeed, this iterative approach leads to the converging results in all studied cases.

As an example, we consider the initial real-valued hexagonal profile and generate the corresponding imaginary profile following a directionality field in the form of a sink, the real, imaginary part and Fourier transform of the profile are provided on the first row of Fig. 5. To be able to realize such complex profile with available materials, we apply local HT iteratively to restrain the complex index values within physical limits. The results for different restriction dimensionalities are shown in Fig. 3(b-d). The index values in complex space illustrate that the iterative procedure precisely limits the refractive indices within the designated area, ring or a set of points on a ring, see the third column in Fig. 5. The corresponding density distributions of complex index, plotted as an inset, also show the spreading of restricted values inside the desired bounds. Note that there are many possible ways to restrict the complex indices during the iterative procedure beyond the ones here provided. In addition, the procedure is independent of the background potential profile and can be applied to arbitrary initial distribution i.e. quasiperiodic, random or localized. Moreover, it is also possible to achieve analogous directionality fields with only losses, i. e., eluding gain, thus increasing the feasibility for practical realization. B. Finally, the robustness and convergence of the iterative procedure was also studied under different restrictions showing that, for the considered restrictions, it remains the same regardless of background pattern while the when the restriction area shrinks requires more iterations. In the square case, the large restriction area leads to a fast converge, as compared to ring and discrete cases [8].

a)

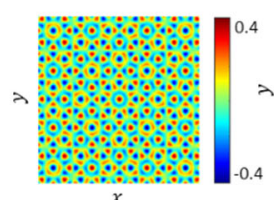

b)

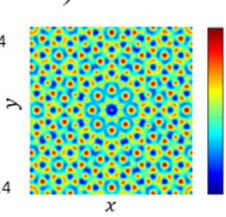

c)

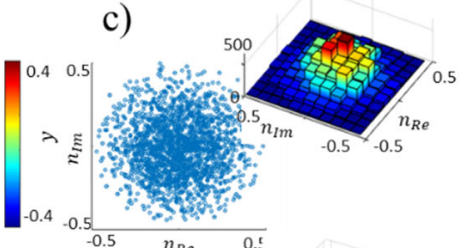

d)

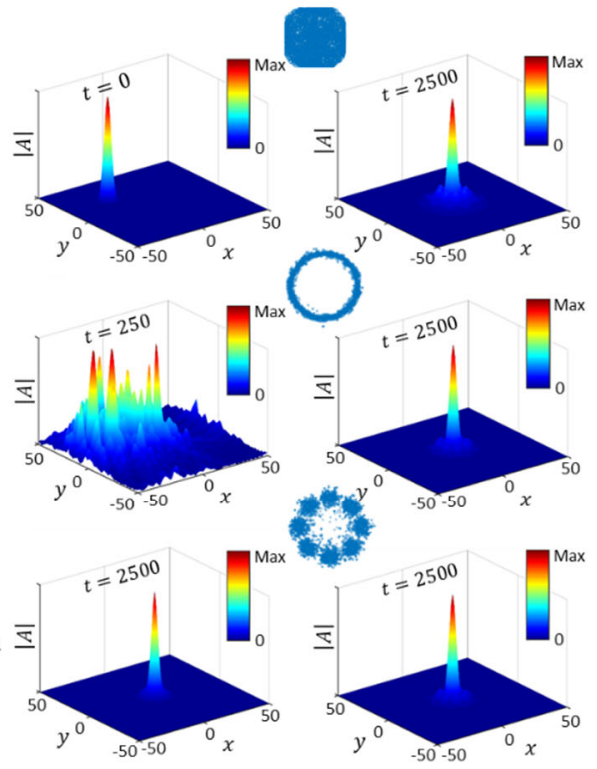

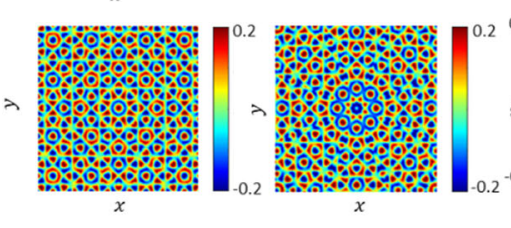
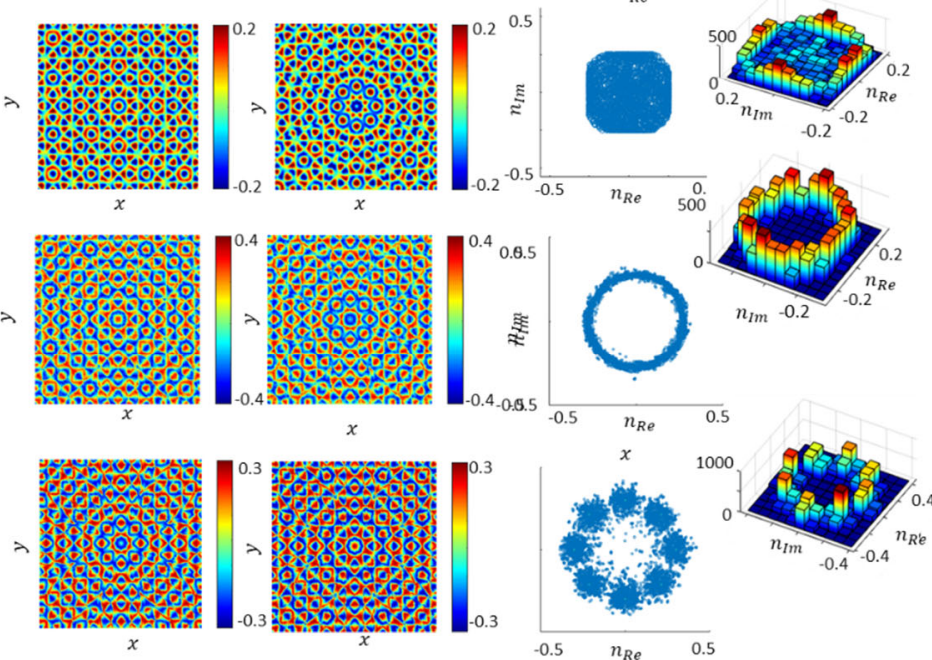

$\begin{array}{lll}-0.5 & n_{R e} & 0.5\end{array}$

FIG. 5. Complex refractive index distributions for sink directionality: (a)/(b) real/imaginary profiles of the HT of an hexagonal background pattern, (c) dimensionality restriction local HT. In restricted HT cases, the complex index profiles are obtained after fifteen iterations. In each row we provide the dimensional restrictions illustrated in Fig. 4, : 2D $\rightarrow 2 \mathrm{D}$ (square), $2 \mathrm{D} \rightarrow 1 \mathrm{D}$ (ring) and $2 \mathrm{D} \rightarrow 0 \mathrm{D}$ (set of discrete points). (d) Field evolution in the modified HT media for a sink directionality. The system is excited with a gaussian beam placed at an arbitrary position within the structure as shown in 
the plot corresponding to $t=0$. The numerically calculated transient and steady state final localized states are provided for each dimensionality restriction.

Next, to verify the directionality effect in restricted HT, we perform numerical simulations using the Schrödinger equation (for paraxial optics) for linear systems with given complex vectorial potentials. Similar results are obtained with full wave simulations, demonstrating three validity of the proposal beyond the paraxial approximation. We observe that the constraints have a weak influence on the pattern of potential flows with reference to sink, as shown in the computed results. The simulated steady-state field distributions using paraxial equation to validate the "Restricted Hilbert transform" proposal expressed are provided in the last column of Fig. 5. A gaussian source is initially placed at an arbitrary position within the modified structure, and after the initial transients the field is efficiently concentrated around the designed sink, placed at center. The final localized states for different restrictions are a result of the field of directionality.

\section{POTENTIAL APPLICATIONS OF THE RESTRICTED HT}

In fact, the restricted HT may find direct applications in some application of the HT that have already been theoretically explored but not yet experimentally demonstrated. This is the case of the regularization of the emission of semiconductor light sources. A non-Hermitian potential in the form of a sink, as in Fig. 1.c, was proposed to be implemented in VCSELS, as shown in Fig. 6a. A radially shifted periodic refractive index and gain-loss modulations accumulate the generated light from the entire active layer and concentrate it around the structure axis to emit narrow, bright beams. The unstable spatiotemporal dynamics, shown on the left image of Fig. 6b, is spatially regularized and stabilized due to asymmetric inward radial coupling between transverse wave vectors. The effect occurs for particular phase differences of the radial refractive index and gain-loss modulations as depicted in the central image of Fig. $6 \mathrm{~b}$ where the main concentration is obtained close to $90^{\circ}$. Also, a non-Hermitian configuration controls and stabilize the spatiotemporal dynamics of edge emitting lasers. A pump modulation, with a mirror-symmetric central axis which induces in-phase gain and refractive index modulations due to the Henry factor. Both modulations may be, in turn, spatially shifted by an appropriate index profile to yield to a local PT symmetry within the modified BAS laser. Such local PT-symmetry potential induces an inward mode coupling, accumulating the light generated from the entire active layer at the central symmetry axis, which ensures spatial regularization and temporal stability, a central symmetry axis enhances and localizes the generated light 10], see Fig. 6b. An analogous scheme may be extended extend the mirror-symmetric non-Hermitian coupling to an array of EEL (diode bar) to achieve asymmetric coupling not within a single laser but between neighboring lasers. The non-Hermitian EEL bar scheme takes advantage of the symmetry coupling breaking, achieving a localized and central output that could directly connect to optical fibers [10] [11].

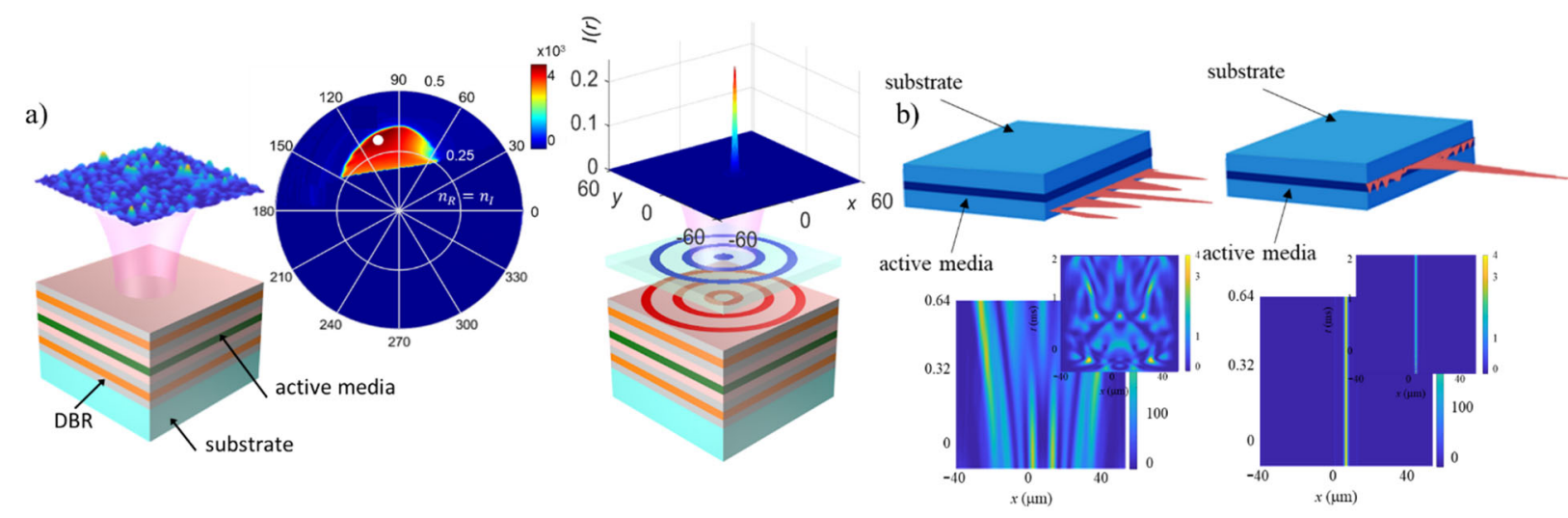

Figure 6. Scattering cancellation applications in linear and nonlinear media. a) Scattering from a dielectric S-shaped object, and broadband invisibility from the modified by the global Hilbert transform with a symmetric kernel. The insets show the permittivity. b)/c) Regularization of the radiation from a VCSEL/edge emitting laser. 


\section{CONCLUSIONS}

To conclude, we show that the proposed restricted Hilbert transform may have applications the generation of feasible systems holding local PT-symmetry to manage arbitrary field flows in higher dimensional non-Hermitian systems under a realistic parameter space. The studied restrictions involve reducing the dimensionaly from $2 \mathrm{D}$ to $1 \mathrm{D}$ and $0 \mathrm{D}$ to achieve the refractive indices within desired ranges. The procedure provides a substantial control over the chosen index values as compared to conventional generalized HT transform following arbitrary vector fileds or the homogeneous spatial KramersKronig relation. The calculated index profiles could be experimentally realized with limited collection of realistic materials by locally tuning the real and imaginary part of dielectric media. Moreover, the discrete HT transform requires minimum three different materials, three unaligned points in susceptibility complex plane for realization [12]. It also offers a general design strategy to implement any desired field configuration in a broad class of non-Hermitian systems conceptually different from existing coordinate transformation approaches. We believe that the proposal opens new possibilities to practical realizations to control the wave dynamics in semiconductor lasers or other linear and nonlinear physical systems based on engineered HT media with realistic index and gain/loss values.

We acknowledge the financial support of Spanish Ministerio de Ciencia e Innovación (PID2019-109175GB-C21). Research Council of Lithuiania (LMTLT), and King Abdullah University of Science and Technology (KAUST) Office of Sponsored Research (OSR) under Award No. OSR-2016-CRG5-2950, KAUST Baseline Research Fund BAS/1/1626-0101, and by NATO SPS research grant No: 985048 .

\section{REFERENCES}

[1] M. Botey, R. Herrero, K. Staliunas, Light in materials with periodic gain-loss modulation on a wavelength scale, Physical Review A 82, 013828, (2010).

[2] C. M. Bender and S. Boettcher, Real Spectra in Non-Hermitian Hamiltonians Having PT SymmetryPhysical Review Letters 80, 5243 (1998).

[3] R. El-Ganainy, K. G. Makris, M. Khajavikhan, Z. H. Musslimani, S. Rotter and D. N. Christodoulides, NonHermitian physics and PT symmetry, Nature Physics 14, 4323, (2017).

[4] W. W. Ahmed, R. Herrero, M. Botey, Z. Hayran, H. Kurt, K. Staliunas. Directionality fields generated by a local Hilbert transform. Physical Review A: 033824, (2018).

[5] S. A. R. Horsley, C. G. King, and T. G. Philbin, Wave propagation in complex coordinates, J. Opt. 18, 044016 (2016).

[6] S. Longhi, Wave reflection in dielectric media obeying spatial Kramers-Kronig relations, Europhys. Lett. 112, 64001 (2015).

[7] Z. Hayran, R. Herrero, M. Botey, H. Kurt, K. Staliunas Invisibility on demand based on a generalized Hilbert transform. Phys. Rev. A. 98(1):013822, 2018. Z. Hayran, H. Kurt, R. Herrero, M. Botey, K. Staliunas. Alldielectric self-cloaked structures. ACS photonics 5(5): 2068-73, (2018).

[8] W. W. Ahmed and R. Herrero and M. Botey and Y. Wu and K. Staliunas, Restricted Hilbert transform for nonHermitian management of fields, Physical Review Applied, 14(4), 044010, (2020).

[9] W. W. Ahmed, R. Herrero, M. Botey, Y. Wu, K. Staliunas, Regularization of vertical-cavity surface-emitting laser emission by periodic non-Hermitian potentials. Optics Letters 44, 3948-51, (2019).

[10] J. Medina, R. Herrero, M. Botey, K. Staliunas, Stabilized narrow beam emission from broad area semiconductor lasers, Physical Review A, 101, 033833, (2020).

[11] J. Medina, R. Herrero, M. Botey, K. Staliunas, Non-Hermitian arrangement for stable semiconductor laser arrays, accepted in Optics Express, (2021).

[12] W. W. Ahmed, R. Herrero, M. Botey, Y. Wu, K. Staliunas, Inverse-design of non-Hermitian potentials for ondemand asymmetric reflectivity. Optics Express, 29(11), 17001-17010. (2021). 